A LETTERS JOURNAL EXPLORING THE FRONTIERS OF PHYSICS

OFFPRINT

\title{
Bistability and hysteresis in dense shear granular flow
}

\author{
E. KHAIN
}

EPL, 87 (2009) 14001

Please visit the new website www.epl journal.org 


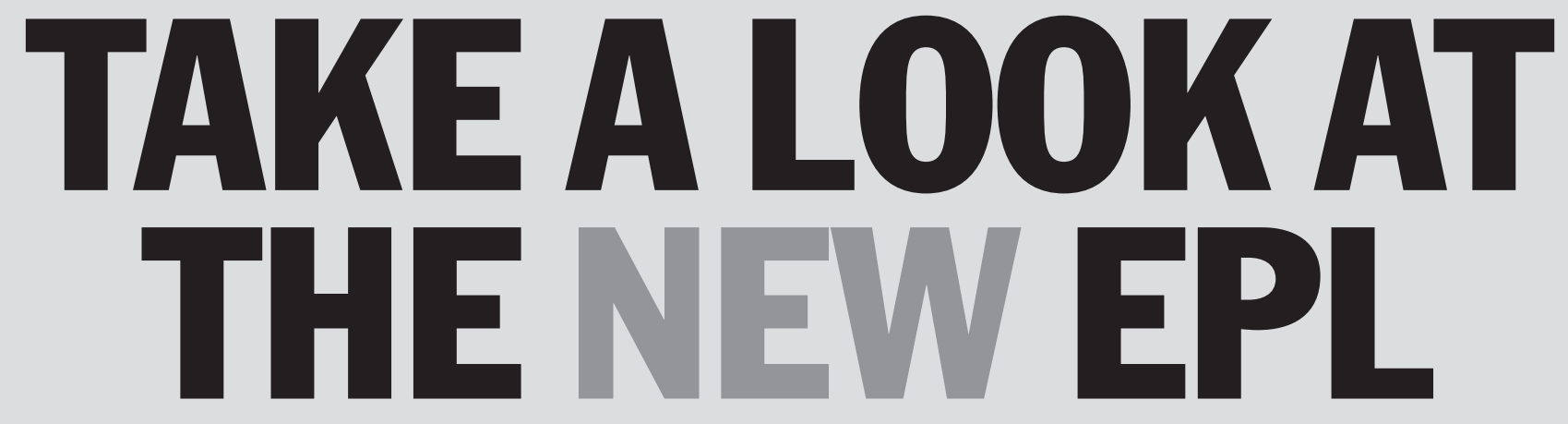

\section{Europhysics Letters (EPL) has a new online home at www.epljournal.org}

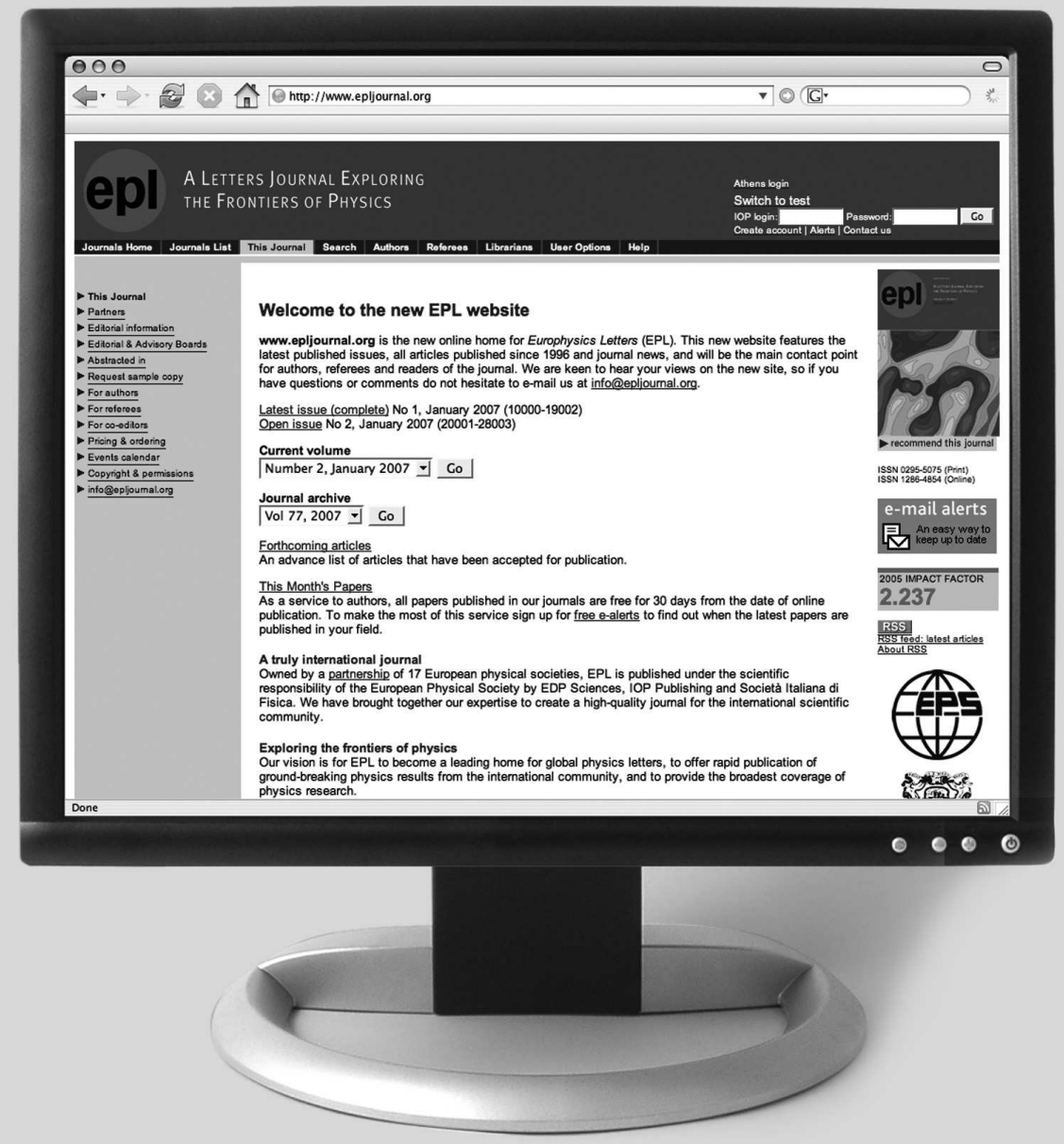

Take a look for the latest journal news and information on:

- reading the latest articles, free!

- receiving free e-mail alerts

- submitting your work to EPL 


\title{
Bistability and hysteresis in dense shear granular flow
}

E. $\operatorname{KHAIN}^{(a)}$

Department of Physics, Oakland University - Rochester, MI 48309, USA

received 26 December 2008; accepted in final form 14 June 2009 published online 15 July 2009

PACS $45.70 . \mathrm{Mg}$ - Granular flow: mixing, segregation and stratification

PACS 45.70.Qj - Pattern formation

PACS 83.50.Ax - Steady shear flows, viscometric flow

\begin{abstract}
We consider dense Couette flow of inelastically colliding hard disks. Depending on the inelasticity of particle collisions and average density of particles, various flow regimes are found, and possible selection mechanisms are discussed. These regimes are investigated using both hydrodynamic equations and event-driven molecular dynamics (MD) simulations. One of the most intriguing flow regimes is the "plug flow", where a solid-like cluster coexists with a fluid layer. The entire phase diagram of parameters is explored, and bifurcation diagrams are presented. We theoretically predict bistability and hysteretic behavior and confirm this prediction in MD simulations.
\end{abstract}

Copyright (C) EPLA, 2009

Introduction. - Fluidized granular matter consists of a huge number of macroscopic particles that move and collide inelastically. Due to inelastic collisions between particles, one needs to pump energy into the system in order to maintain the particles motion. As a result, a driven granular system can achieve a steady state. These steady states are intrinsically far from equilibrium, since there is a (constant) heat flux through the system: the energy pumped into the system is lost in inelastic collisions. Therefore, driven granular media provides an exciting example of far from equilibrium systems [1].

There are several ways to pump energy into the granular media. One way is to vibrate the system (either from below or by a side wall) [2]. We consider here another mechanism of driving: pump energy by shear [3]. We focus on rapid dense shear granular flow and analyze various non-equilibrium steady states (different steady flow regimes) and transitions between them, exploring the entire phase diagram of parameters. The dynamics of this system is highly non-linear, so it is not surprising that two (or more) steady states may exist for the same set of parameters. This type of phenomena have been observed experimentally [4] but, to our knowledge, they have never been addressed theoretically in driven granular systems. Here, for the first time, we report on phenomena of bistability and hysteresis in a dense shear granular flow.

Dense granular flows have recently attracted a significant attention [3]. Different regimes of dense flows were

\footnotetext{
(a) E-mail: khain@oakland.edu
}

investigated experimentally, theoretically, and using molecular dynamics (MD) simulations. One of the most intriguing flow regimes is the "plug flow" [5], which resembles the phenomenon of shear banding [6]. In this regime, a solidlike layer of particles coexists with (one or two) fluid-like layers [5,7-9], see fig. 1 (the upper panel). While in the fluid layer the flow velocity varies across the layer, the solid-like cluster moves as a whole with some constant velocity. This flow regime was observed in MD simulations, but only recently its theoretical explanation has been proposed [7]. It was found [10] that the shear viscosity of hard-disks fluid diverges not at the density of crystalline close packing $n_{\max }=2 /\left(\sqrt{3} d^{2}\right)$ (where pressure, inelastic heat losses and thermal conductivity diverge), but at a smaller density, $n_{\eta}<n_{\max }$. As a result, the velocity does not vary across the "solid" cluster ( since $n_{\eta}<n<n_{\max }$ ), while the density and the temperature do vary. The lower panel of fig. 1 shows a magnified picture of the crystallized region inside the cluster. Although the particles form an ordered structure, the density is everywhere smaller than that of the maximal dense crystalline packing, $n_{\max }$. The granulate is fluidized, the particles are not at rest, and the granular temperature is larger than zero.

In this work, we investigate different regimes of a dense fluidized granular flow and present the entire phase diagram of parameters. We use two different approaches: molecular dynamics (MD) simulations [11] and NavierStokes granular hydrodynamics [12]. In order to describe very dense flows, one needs to choose specific constitutive relations, which are valid also in the dense limit (see ref. [8] 


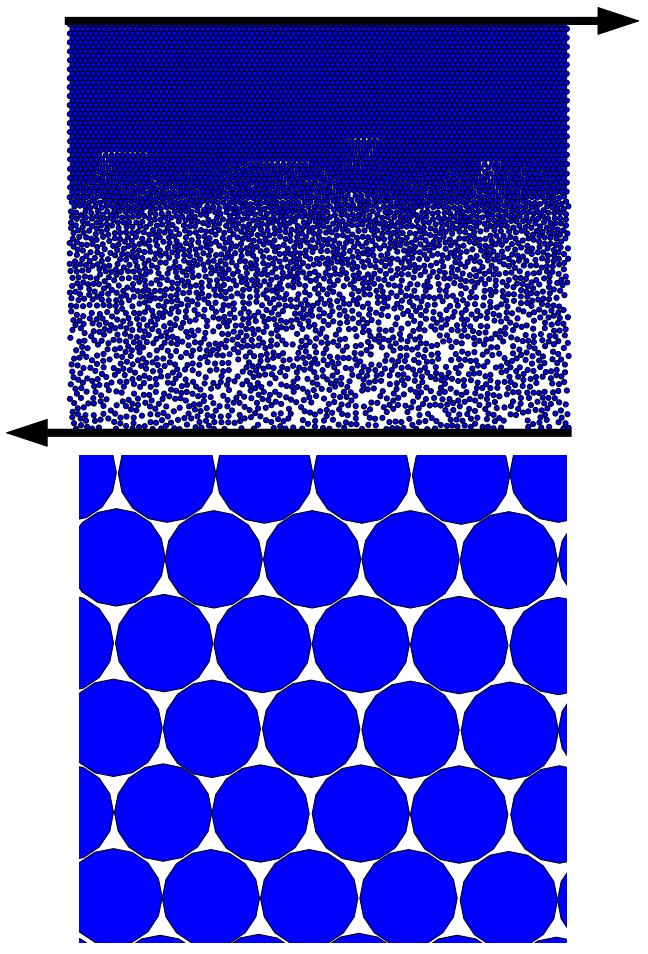

Fig. 1: (Colour on-line) Snapshot of the two-phase flow for $r=$ 0.992, $N=6480$, and $\langle n\rangle=0.7244(\langle\nu\rangle=0.657)$ (upper panel). The corresponding density, temperature and velocity profiles are shown in fig. 2. The lower panel shows a magnified picture of the crystallized region inside the cluster. The granulate is fluidized, there are empty spaces between particles, the density is smaller than that of close packing, the granular temperature is larger than zero. However, since $n_{\eta}<n$, the horizontal flow velocity does not vary across the cluster.

and references therein for details). We use the constitutive relations proposed in ref. [8], which incorporate the viscosity divergence. The results of hydrodynamic analysis are compared with the results of MD simulations.

The rest of the paper is organized as follows. First, we present the model and formulate a hydrodynamic approach. Then we describe the plug flow regime. Finally, we present the entire phase diagram and address the phenomenon of bistability and hysteresis. We conclude with a summary and discussion of the results.

Model. - We consider the dynamics of a system of $N$ inelastically colliding hard disks driven by two opposite walls, located at $y=H / 2$ (the "upper" wall) and at $y=$ $-H / 2$ (the "lower" wall). The two walls move in the $x$-direction with opposite velocities (the upper panel of fig. 1), $u_{0}$ and $-u_{0}$ respectively. There is no gravity in the model. The inelasticity of collisions is described by the normal restitution coefficient, $r$. We assume the limit of nearly elastic collisions, $1-r^{2} \ll 1$. Boundary conditions in the $x$-direction are periodic, while in the $y$-direction we demand no-flux and no-slip boundary conditions. To implement these boundary conditions in MD simulations, we use the following procedure. Upon a collision with a driving wall, the normal particle velocity switches sign, while the new tangential velocity component is taken from Maxwell-Boltzmann distribution. The mean velocity of this distribution equals the wall velocity, and the variance corresponds to the instant temperature of the layer next to the wall. The particles have unit mass and diameter $d$. Initially, particles have random velocities and are distributed homogeneously over the system.

To describe different flow regimes theoretically, we use Navier-Stokes granular hydrodynamics, which deals with coarse-grained quantities: number density of grains, $n(\mathbf{r}, t)$, granular temperature $T(\mathbf{r}, t)$, and mean flow velocity $v(\mathbf{r}, t)[13]$ :

$$
\begin{aligned}
& \mathrm{d} n / \mathrm{d} t+n \boldsymbol{\nabla} \cdot \mathbf{v}=0, \\
& n(\mathrm{~d} \mathbf{v} / \mathrm{d} t)=\boldsymbol{\nabla} \cdot \mathbf{P}, \\
& n(\mathrm{~d} T / \mathrm{d} t)=-\boldsymbol{\nabla} \cdot \mathbf{Q}+\mathbf{P}: \boldsymbol{\nabla} \mathbf{v}-\Gamma
\end{aligned}
$$

Here $\mathbf{P}$ is the stress tensor, $\mathbf{Q}$ is the heat flux, and $\Gamma$ is inelastic heat losses. The stress tensor $\mathbf{P}$ is given by $\mathbf{P}=[-p(n, T)+\mu(n, T) \operatorname{tr}(\mathbf{D})] \mathbf{I}+2 \eta(n, T) \hat{\mathbf{D}}$, where $\mathbf{D}=$ $(1 / 2)\left[\boldsymbol{\nabla} v+(\boldsymbol{\nabla} v)^{T}\right]$ is the rate of deformation tensor, $\hat{\mathbf{D}}=$ $\mathbf{D}-\frac{1}{2} \operatorname{tr}(\mathbf{D}) \mathbf{I}$ is the deviatoric part of $\mathbf{D}$, and $\mathbf{I}$ is the identity tensor, $\eta(n, T)$ and $\mu(n, T)$ are the shear and bulk viscosities. The heat flux $\mathbf{Q}$ is given by $\mathbf{Q}=-\kappa(n, T) \nabla T$, where $\kappa(n, T)$ is the coefficient of thermal conductivity. In this study, we consider the limit of nearly elastic collisions, $1-r^{2} \ll 1$. In this limit, we can safely neglect an additional term in the expression for the heat flux, which is proportional to the density gradient [14]. We also neglect inelastic corrections to transport coefficients [14] and replace $1-r^{2}$ factor in the inelastic heat losses term by $2(1-r)$.

For dilute and moderately dense flows, the Enskoglike shear viscosity, $\eta_{E}(n, T)$, the thermal conductivity $\kappa_{E}(n, T)$, the inelastic heat losses $\Gamma_{E}(n, T)$, and the equation of state $p_{E}$

$$
\begin{aligned}
& \eta_{E}=\frac{4 \nu T^{1 / 2} G_{E}}{\pi^{3 / 2} d}\left[1+\frac{\pi}{8}\left(1+\frac{1}{G_{E}}\right)^{2}\right], \\
& \kappa_{E}=\frac{8 \nu T^{1 / 2} G_{E}}{\pi^{3 / 2} d}\left[1+\frac{9 \pi}{16}\left(1+\frac{2}{3 G_{E}}\right)^{2}\right], \\
& \Gamma_{E}=\frac{8(1-r) n T^{3 / 2} G_{E}}{\pi^{1 / 2} d} \\
& p_{E}=n T\left(1+2 G_{E}\right)
\end{aligned}
$$

are in a reasonable agreement with the results of eventdriven MD simulations [15]. Here $G_{E}=\nu(1-7 \nu / 16) /$ $(1-\nu)^{2}, \nu=n\left(\pi d^{2} / 4\right)$ is the solid fraction, and index $E$ stays for "Enskog". For very large densities, one can use free volume arguments and show that all constitutive relations (except for shear viscosity) diverge at the density of close packing. In a recent work [8], the interpolation of constitutive relations between the dilute and dense limits was proposed (see also refs. $[10,16]$ ) and tested in a series 
of MD simulations; a viscosity divergence was also taken into account. The modified constitutive relations are

$$
\begin{aligned}
& p=n T(1+2 G), \\
& \Gamma=\frac{8(1-r) n T^{3 / 2} G}{\pi^{1 / 2} d}, \\
& \kappa=\kappa_{E}\left(1+0.1 \nu-10 \nu^{10}+\frac{0.11}{\nu_{\max }-\nu}-\frac{0.11}{\nu_{\max }}\right), \\
& \eta=\eta_{E}\left[1+a_{\eta}\left(\nu / \nu_{\eta}\right)^{3} /\left(\nu_{\eta}-\nu\right)\right],
\end{aligned}
$$

where $G=G_{E}+\theta\left[\nu_{\max } /\left(\nu_{\max }-\nu\right)-G_{E}\right]$. Here, $\theta$ is the interpolation function, given by $\theta=[1+\exp$ $\left.\left(\left(\nu_{c}-\nu\right) / m_{0}\right)\right]^{-1}$ with $\nu_{c}=0.70, m_{0}=0.0111, \nu_{\eta}=0.71$, $a_{\eta}=0.037$, and $\nu_{\max }=\pi /(2 \sqrt{3})[10,16]$. Notice that the pressure (the first equation in eqs. (3)) is a monotonically increasing function of density.

Plug flow. - The simplest steady granular Couette flow is a uniform shear flow (USF), where the velocity gradient, the temperature, and the density are constants. When the inelasticity of particle collisions becomes sufficiently large, the USF loses its stability [17], which leads to density inhomogeneities in the system. If the average density is sufficiently high, there will be regions where the density is higher than the density of viscosity divergence, $n>n_{\eta}$. The granulate in this region behaves like a solid cluster; in the neighboring regions, where the density is smaller, $n<n_{\eta}$, it shows the usual fluid-like behavior. Figure 1 shows an example of this solid-fluid coexistence, as found in MD simulations of $N=6480$ inelastic hard disks. Initially, the particles were distributed homogeneously over the system. In order to make sure that the system reaches the steady state, we followed the time dependence of $y$-component of center of mass of the system, making sure that after a short transient, $y_{\mathrm{cm}}$ reaches a plato and does not change anymore. A solid cluster next to one of the walls moves as a whole with the velocity, which approximately equals the velocity of the wall.

Consider a steady solution $(\partial / \partial t=0)$ of eqs. (1), assuming flow in $x$-direction, zero vertical velocity and $\partial / \partial x=0$. We measure the coordinate $y$ in units of the system height $H$, the horizontal velocity $u$ in units of the wall velocity $u_{0}$, the temperature $T$ in units of $u_{0}^{2}$, the density $n$ in units of $n_{\max }$, the pressure $P$ in units of $n_{\max } u_{0}^{2}$. Then eqs. (1) reduce to:

$$
\begin{aligned}
& \frac{\mathrm{d}}{\mathrm{d} y}\left(f_{2} T^{1 / 2} \frac{\mathrm{d} u}{\mathrm{~d} y}\right)=0 \\
& \frac{\mathrm{d}}{\mathrm{d} y}\left(f_{1} T^{1 / 2} \frac{\mathrm{d} T}{\mathrm{~d} y}\right)+\frac{f_{2} T^{1 / 2}}{4}\left(\frac{\mathrm{d} u}{\mathrm{~d} y}\right)^{2}-R f_{3} T^{3 / 2}=0 \\
& f_{4} T=\text { const }
\end{aligned}
$$

where $R=(16 / \pi)(1-r)(H / d)^{2}$ is the hydrodynamic heat loss parameter, and the functions $f_{i}$ are the
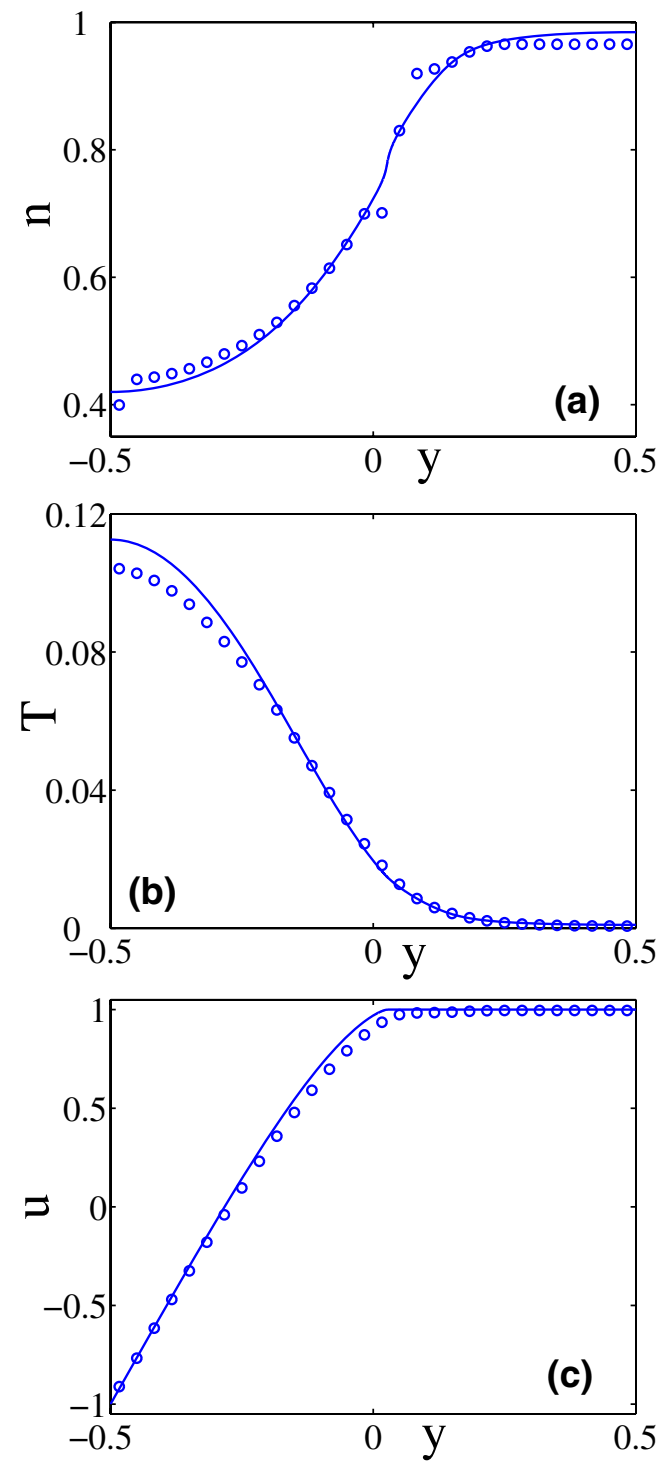

Fig. 2: (Colour on-line) The density (a), temperature (b), and velocity (c) profiles of the two-phase flow for $R=259.07$ and $\langle n\rangle=0.7244(\langle\nu\rangle=0.657)$. The results of MD simulations are shown by circles; solid lines denote the theoretical curves obtained from numerical solution of eqs. (4). The corresponding snapshot of the system is shown in fig. 1.

density-dependent parts of the constitutive relations (eqs. (3)):

$$
\begin{aligned}
& f_{1}=\kappa(\sqrt{\pi} d / 2) T^{-1 / 2}, \\
& f_{2}=\eta(2 d \sqrt{\pi}) T^{-1 / 2}, \\
& f_{3}=\nu G, \\
& f_{4}=n(1+2 G) .
\end{aligned}
$$

We demand no-flux and no-slip boundary conditions: $\mathrm{d} T / \mathrm{d} y(y=-1 / 2)=\mathrm{d} T / \mathrm{d} y(y=1 / 2)=0, \quad u(y=-1 / 2)=$ $-1, u(y=1 / 2)=1$. Finally, the conservation of the total number of particles is given by $\int_{-1 / 2}^{1 / 2} n(y) \mathrm{d} y=\langle\nu\rangle / \nu_{\max }$. Figure 2 shows the profiles of density (the upper panel), 
temperature (the middle panel), and horizontal velocity (the lower panel). The hydrodynamic profiles (solid lines) are computed from eqs. (4). The numerical procedure is similar to that described in refs. [7,8]: eqs. (4) are solved for each layer separately, demanding continuity of density, heat flux, and velocity at the interface between the layers. For the solid-like layer (where $n>n_{\eta}$ ), the first equation of eqs. (4) is replaced by $u=$ const. In general, the value of this constant should be found from the overall solution. In case when a cluster is next to one of the walls, its velocity equals the velocity of the corresponding wall, $u=1$ (see fig. $2(\mathrm{c})$ ). We found these two-layer solutions of eqs. (4) using a numerical shooting procedure [7]. Note that the temperature and density vary across the cluster, but the velocity is constant. This is because at such high densities the shear viscosity has diverged, but thermal conductivity, pressure and inelastic heat losses are finite.

To test the hydrodynamic predictions, we performed MD simulations. Overall, the agreement between the hydrodynamic profiles (fig. 2, solid lines) and the profiles calculated from MD simulations (fig. 2, circles) is good. However, one can see small discrepancies (less than 10 percent), which are more visible for the temperature profile. Although for the $T(y)$ these discrepancies occur near one of the walls, we do not believe they are caused by the boundary conditions implemented in MD simulations. The origin for the 10-percent errors is most likely in the hydrodynamic constitutive relations. Although the theoretical formulas for pressure, heat losses and viscosity were verified in MD simulations in a wide range of densities $[8,10,16]$ in various systems and settings, the data for coefficient of thermal conductivity, $\kappa$, at high densities is not sufficient, so the formula for $\kappa$ [8] (fitted to the available data [10]) might be the reason for 10 percent discrepancy [18].

Phase diagram, bistability and hysteresis. - Let us explore the entire phase diagram of parameters, examining what flow regimes are possible for different values of $R$ and $\langle\nu\rangle$. It is well known that the USF becomes unstable for sufficiently large inelasticity of particle collisions [17]. The instability thresholds can be written in the following form [8]:

$$
R_{c}=\pi^{2} m^{2} f_{1}\left[\frac{f_{4}\left(\mathrm{~d} f_{3} / \mathrm{d} \nu\right)}{\left(\mathrm{d} f_{4} / \mathrm{d} \nu\right)}+\frac{f_{4} f_{3}\left(\mathrm{~d} f_{2} / \mathrm{d} \nu\right)}{f_{2}\left(\mathrm{~d} f_{4} / \mathrm{d} \nu\right)}-2 f_{3}\right]^{-1}
$$

where functions $f_{i}$ are given by eqs. (5), and $m$ is the mode number (the wave number of perturbation is $\left.k_{m}=m \pi\right)^{1}$. The two curves corresponding to $m=1$ and $m=2$ are shown in fig. 3(a) (lower and upper dashdotted lines, respectively). Figure 3(a) presents the phase diagram of the system. The curves presented on this diagram are computed theoretically, two solid lines and two dashed lines (computed from eqs. (4)) divide the

\footnotetext{
${ }^{1}$ This instability threshold coincides with the instability criteria obtained in refs. [9,17].
}
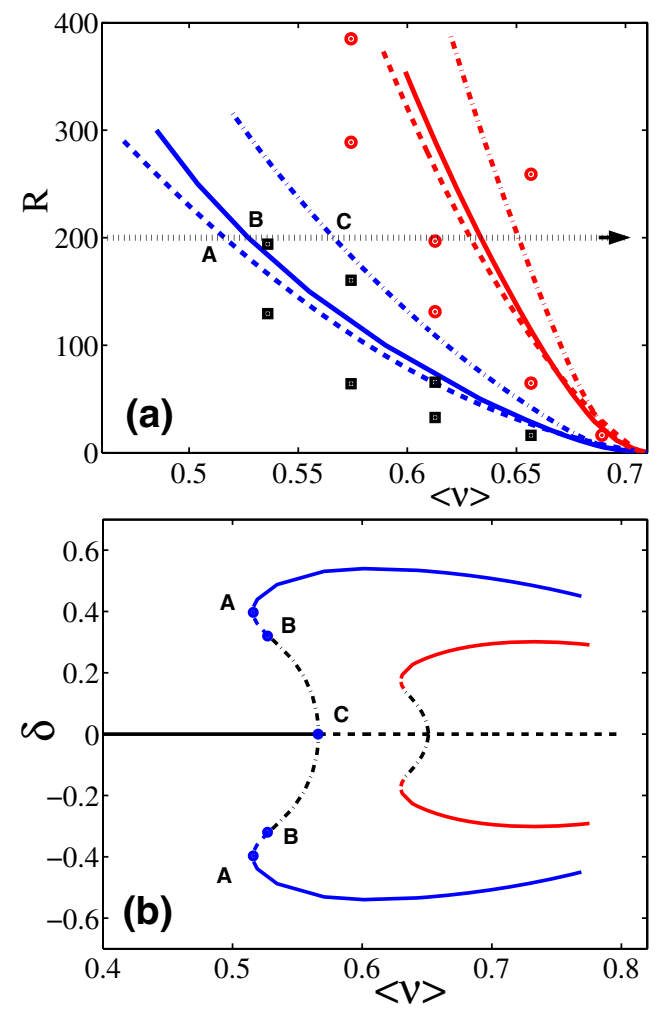

Fig. 3: (Colour on-line) Phase diagram (a) and bifurcation diagram (b) of dense fluidized shear granular flow. Phase diagram (a): symbols denote the results of MD simulations, USF solutions are denoted by squares, asymmetric plug flow solutions are denoted by circles. Six theoretical curves divide the diagram into many regions; in each region a different set of solutions exist (see text). The two dash-dotted lines denote the instability thresholds of the USF with respect to perturbations with $m=1$ and $m=2$, respectively. The bifurcation diagram (b) shows the density contrast $\delta$ vs. $\langle\nu\rangle$ for different steady flow solutions at a fixed heat loss parameter, $R=200$. It is obtained by crossing the phase diagram by the horizontal dotted arrow (see text). The branches on the left (points A, B, C) denote asymmetric solutions $(m=1)$ and correspond to the three lowest curves in (a), the branches on the right denote symmetric solutions $(m=2)$ and correspond to the three upper curves in (a).

diagram into many regions; in each region a different set of solutions exist (see below). Symbols show the results of MD simulations: USF solutions are denoted by squares, while asymmetric plug flow solutions (with cluster next to one of the walls) are denoted by circles. To explain the meaning of the curves, let us consider the bifurcation diagram, fig. 3(b). We define $\delta$ as the density contrast in the system. For $m=1, \delta$ is the density difference between the two walls, $\delta \equiv n(y=1 / 2)-n(y=-1 / 2)$, for $m=2, \delta$ is the density contrast between the walls and the center of the system, $\delta \equiv n(y=1 / 2)-n(y=0)$. We compute $\delta$ as a function of parameters $R$ and $\langle\nu\rangle$ for different flow regimes from eqs. (4). The three lower curves in fig. 3(a) and the set of low-density branches in fig. 3(b) (CBA branches) correspond to asymmetric solutions, the similar remaining 


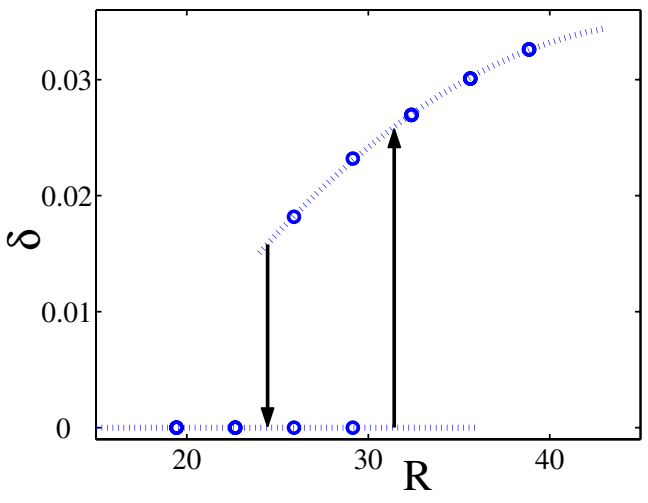

Fig. 4: (Colour on-line) Bistability and hysteresis as observed in MD simulations for $\langle n\rangle=0.7244(\langle\nu\rangle=0.657)$.

set of curves in both figures correspond to symmetric solutions. Note that the branches with $\delta>0$ and $\delta<0$ are identical; for example, for asymmetric plug flow solutions the plug is near the upper wall or near the lower wall, respectively. Let us focus therefore on $\delta \geqslant 0$ branches.

Let us fix $R=200$ and increase the average filling fraction $\langle\nu\rangle$, as shown by the dotted arrow, fig. 3(a). For sufficiently small $\langle\nu\rangle$, the only possible solution is the USF; it is stable (a solid line, $\delta=0$, in fig. 3(b)). Increasing $\langle\nu\rangle$, we cross the dashed line (point A), and two new solutions appear (point $\mathrm{A}, \delta \geqslant 0$ branches, fig. 3(b)), these are two plug flow solutions. The solution with the larger $\delta$ seems to be stable, while the one corresponding to the smaller $\delta$ is unstable. As we increase $\langle\nu\rangle$ further on, we cross the solid line in fig. 3(a) (point B). Here (point B, fig. 3(b) the unstable plug flow solution turns to the fluid-like solution with non-linear density, temperature and velocity profiles (a similar symmetric solution was described in [7], this solution seems to be unstable as well). When $\langle\nu\rangle$ is further increased, the dotted arrow in fig. 3(a) crosses the dash-dotted line, point C. This dash-dotted line represents the instability threshold for the USF solution (for $m=1$ ). The corresponding point $\mathrm{C}$ in fig. 3(b) shows an inverse pitchfork bifurcation. For larger densities, the USF becomes unstable, so the only stable solution is the (asymmetric) plug flow. When $\langle\nu\rangle$ is further increased, the dotted arrow crosses the curves, corresponding to symmetric solutions. The structure of bifurcation diagram is very similar to that of asymmetric branches.

Our theoretical results show that in the interval of densities $\left(\langle\nu\rangle_{A},\langle\nu\rangle_{C}\right)$, fig. 3(b), there are two stable solutions: a USF solution and a regime of fluid-solid coexistence with a plug near one of the walls. A similar bifurcation diagram can be obtained if we vary $R$, keeping $\langle\nu\rangle$ fixed. In this case, the theory predicts bistability in some interval of heat loss parameters $R$. To test the predictions, we investigated the phenomenon of bistability in MD simulations, see fig. 4. In order to obtain this hysteretic loop, we performed the following procedure. First, we started with the high restitution coefficient, $r$ (small $R$ ) in the region where the USF is the only possible solution. Then we decreased the restitution coefficient, using previously obtained flow configuration (USF solution) as the initial state. For every value of $r$, we verified that a steady state is achieved, by following the center of mass of the system. When $r$ becomes small enough ( $R$ becomes sufficiently large), the initial uniform state jumps to the plug flow regime. The same procedure is performed on the way back, when we started with a plug flow and obtained a series of solutions corresponding to the increasing values of the restitution coefficient (decreasing values of $R$ ). At a certain small enough $R$, plug flow solution does not exist any longer, and the initial state develops into the USF.

Summary and discussion. - In the present study, we analyzed the behavior of a dense fluidized shear granular flow. Depending on the governing parameters, different steady flow regimes were obtained. We investigated the regime of the "plug flow", where a solid-like cluster coexists with a fluid layer. Importantly, this shear-banding regime is obtained within the framework of a hard-sphere model, without friction, cohesion and long-range interactions. Interestingly, a similar plug flow phenomenon has been observed recently in dense colloidal suspensions [19].

We described the fluid-solid coexistence theoretically, by solving equations of granular hydrodynamics, taking into account the effect of viscosity divergence. We explored the entire phase diagram of parameters, identifying various solutions in different parts of the diagram, and presented the corresponding bifurcation diagrams. Solving hydrodynamic equations, we predicted bistability and hysteretic behavior and confirmed this prediction in MD simulations. For lower densities similar bistabilities were theoretically analyzed in refs. [17].

We found regions in the phase diagram, where several steady flow regimes are possible. Which solution is finally selected? MD simulations show that the selected steady flow corresponds to the minimal mode number, $m=1$, so for no-flux boundary conditions only asymmetric solution with plug near one of the walls is observed. Therefore, the solution of cluster in the center of the system (or the solution of two clusters near the walls) $[5,8]$ is a transient. It is known that in many cases, the state with the "largest wavelength" is chosen. A recent example of this rule of thumb in a granular system is the selection problem in freely cooling granular gas in a channel $[20]$. We checked that in our system this rule is consistent (in several cases) with another mechanism of selection, proposed in ref. [9]. Several sets of constitutive relations were examined [9], in particular, Model D took into account both global equation of state and viscosity divergence. The authors introduced a "dynamic friction" $\beta$, and stated that among many possible steady states, the system chooses one with the minimal value of $\beta$ [9]. Note, however, that the hysteretic behavior discovered in this study (both theoretically and in MD simulations) indicates that the selection mechanism is more complex and depends on the initial conditions. 
The stability of the two-phase flow was analyzed in MD simulations. To examine this question theoretically, a linear stability analysis of the two-phase flow regime needs to be done. This analysis is technically quite challenging for the following reason. The steady two-phase flow presented in fig. 2 can be computed numerically (by solving eqs. (4)), taking advantage of the fact that the interface between the fluid-like layer and the solid-like is flat. The position of the interface depends therefore only on the coordinate $y$ (direction, perpendicular to the walls) and does not depend on the coordinate $x$ (along the flow direction). In order to perform the linear stability analysis of the two-phase flow, one needs to add sinusoidal perturbation along the flow direction. In this case, the position of the interface will be both function of $y$ and $x$. The matching of the solution in fluid-like regions with the solution in solid-like regions becomes very challenging in this case.

A similar mathematical challenge occurs in the analysis of thermal granular convection in sufficiently dense system. The instability threshold for thermal granular convection was theoretically derived for dilute granular gases [21]. However, if the mean density in the system is sufficiently high, the simplest static steady state consists of two phases: an almost densely packed levitating cluster and a hot granular gas below it [22]. Again, the interface between the two phases is flat. However, in some region of parameters, this cluster can be broken, and thermal convection develops. This amazing phenomenon was observed in MD simulations [23] and has been recently observed experimentally [24]. Theoretical investigation of this phenomenon might be a promising direction of future research.

Finally, in this study we assumed a constant coefficient of normal restitution. In reality, the restitution coefficient is velocity-dependent, which leads to effective temperature dependence of the hydrodynamic heat loss parameter $R$. It is known that for freely cooling granular gas this affects the clustering instability: clusters might appear only in the transient regime [25]. However, we do not expect this to occur in the case of driven granular media. Two basic ingredients for the two-phase flow analyzed in this paper are the viscosity divergence at some density $n_{\eta}$ and the instability leading to density inhomogeneities; both of them should be present also for the case of velocitydependent restitution coefficient.

$$
* * *
$$

I thank B. MeERson for fruitful discussions.

\section{REFERENCES}

[1] Pöschel T. and Luding S. (Editors), Granular Gases (Springer, Berlin) 2001; Pöschel T. and BRILliantov N. (Editors), Granular Gas Dynamics (Springer, Berlin) 2003; Aranson I. S. and Tsimring L. S., Rev. Mod. Phys., 78 (2006) 641.

[2] For example, Luding S., Clement E., Rajchenbach J. and Duran J., Europhys. Lett., 36 (1996) 247; NIE
X. B., Ben-Naim E. and Chen S. Y., Europhys. Lett., 51 (2002) 679; Khain E. and Meerson B., Europhys. Lett., 65 (2004) 193; Brey J. J. and Ruiz-Montero M. J., Europhys. Lett., 66 (2004) 805.

[3] For example, Bocquet L., Losert W., Schalk D., Lubensky T. C. and Gollub J. P., Phys. Rev. E, 65 (2002) 011307; Volfson D., Tsimring L. S. and Aranson I. S., Phys. Rev. Lett., 90 (2003) 254301; Tsai J. C., Voth G. A. and Gollub J. P., Phys. Rev. Lett., 91 (2003) 064301; DANiELs K. E. and Behringer R. P., Phys. Rev. Lett., 94 (2005) 168001; Cordero P., Risso D. and Soto R., Physica A, 356 (2005) 54; SAItoh K. and Hayakawa H., Phys. Rev. E, 75 (2007) 021302.

[4] Tsai J. C. and Gollub J. P., Phys. Rev. E, 70 (2004) 031303.

[5] Alam M. and Luding S., Phys. Fluids, 15 (2003) 2298; Alam M., Arakeri V. H., Nott P. R., Goddard J. D. and Herrmann H. J., J. Fluid. Mech., 523 (2005) 277.

[6] Mueth D. M., Debregeas G. F., Karczmar G. S., Eng P. J., Nagel S. R. and Jaeger H. M., Nature, 406 (2000) 385.

[7] Khain E. and Meerson B., Phys. Rev. E, 73 (2006) 061301.

[8] Khain E., Phys. Rev. E, 75 (2007) 051310.

[9] Alam M., Shukla P. and Luding S., J. Fluid Mech., 615 (2008) 293.

[10] Garcia-Rojo R., Luding S. and Brey J. J., Phys. Rev. E, 74 (2006) 061305.

[11] Rapaport D. C., The Art of Molecular Dynamics Simulation (Cambridge University Press, Cambridge) 1995.

[12] Goldhirsch I., Annu. Rev. Fluid Mech., 35 (2003) 267.

[13] HafF P. K., J. Fluid Mech., 134 (1983) 401; JENkins J. T. and Richman M. W., Phys. Fluids, 28 (1985) 3485.

[14] For example, Brey J. J., Moreno F. and Dufty J. W., Phys. Rev. E, 54 (1996) 445; Sela N. and Goldhirsch I., J. Fluid Mech., 361 (1998) 41.

[15] Bizon C., Shattuck M. D., Swift J. B. and Swinney H. L., Phys. Rev. E, 60 (1999) 4340.

[16] Luding S., Phys. Rev. E, 63 (2001) 042201.

[17] For example, Alam M. and Nott P. R., J. Fluid Mech, 377 (1998) 99; Alam M., Arakeri V. H., Nott P. R., Goddard J. D. and Herrmann H. J., J. Fluid. Mech., 523 (2005) 277.

[18] One way to measure the density dependence of $\kappa$ in MD simulations is to find a homogeneous system with a substantial temperature gradient, which is not easy. One possible option here is adding gravity to the system, as in Risso D. and Cordero P., J. Stat. Phys., 82 (1996) 1453.

[19] Isa L., Besseling R. and Poon W. C. K., Phys. Rev. Lett., 98 (2007) 198305.

[20] Meerson B., Fouxon I. and Vilenkin A., Phys. Rev. E, 77 (2008) 021307.

[21] Khain E. and Meerson B., Phys. Rev. E, 67 (2003) 021306.

[22] Meerson B., Poschel T. and Bromberg Y., Phys. Rev. Lett., 91 (2003) 024301.

[23] Isobe M., Phys. Rev. E, 64 (2001) 031304.

[24] Eshuis P., van der Weele K., van der Meer D., Bos R. and Lohse D., Phys. Fluids, 19 (2007) 123301.

[25] Poschel T., Brilliantov N. V. and Schwager T., J. Phys.: Condens. Matter, 17 (2005) S2705. 